\title{
Change Management and PLM Implementation
}

\author{
Kamal Cheballah ${ }^{1}$ and Aurélie Bissay ${ }^{2}$ \\ ${ }^{1}$ Audros Technology, PLM Software editor, Lyon, France \\ ${ }^{2}$ GreenLight-IT, Private research center in Informatics, Lyon, France \\ kcheballah@audros.fr, abissay@greenlight-it.fr
}

\begin{abstract}
To capitalize from the management of a company's expertise is reliant on the ability manipulate the data, information, documents, and knowledge that represent these expertise. This process is complex and so to reach this target we need to introduce new tools and methods of work to the company. These changes will face a certain amount of resistance and so attempts have been made by numerous scientific researchers, to introduce new change management methods to minimise this resistance. The classical methods of change management are a $<<$ top-down $>>$ approach which is not really appropriate. Our work proposes a new $<<$ bottom-up $>>$ approach which derives from the users instinctive ability to use certain interface elements.
\end{abstract}

Keywords: Change management, PLM implementation, AudrosBox, Collaborative features.

\section{Introduction}

Companies operate in an environment where it is necessary to be as competitive as possible. This race towards competitiveness can be conducted in different ways, through company strategy, its internal resources, its organization, etc. Our research focuses particularly on the organizational aspects of the company particularly through the information system. Companies' expertise Is an essential element in competitiveness. The focus is on the management of its documents, data, information, and knowledge. This management is the key point in increasing the enterprise productivity. The use of all the tools needed by the fact we need to manage these information have as a consequence a modification if working methods for the users. We contribute through our research by introducing new tools which gives a more progressive way to manage all these information, avoiding the risk of rejecting the system by users

\section{Information System and Change Management}

\subsection{Problematic}

The current situation no longer allows some companies whatever their size to manage their individual expertise. Information, data, knowledge are all principal elements of the company that need to be preserved. 
The entire expertise is often capitalized individually wick renders it impractical for the company. Furthermore its organization and its scalability is directly related to the wishes of those who want to capitalize. This leads to a bottleneck in the execution process of work done in the company. Some players are no longer able to respond to the number of inquiries needed for the execution process.

Document management systems and, PLM, responds to these needs. They aim to organize and manage information and documents within an organization. These systems implement procurement methods, Indexing, classification, management and storage, access and retrieval of data and documents. They therefore contribute to the collaborative work processes, capitalization and the exchange of information.

Management expertise leads to gains and a rapid return on investment for organizations. In this collaborative approach around a software system, it is the effective implementation of the methodology which is the source of success and not just the product which serves only to support this method. To be carried out well, this type of project requires that we dedicate a support, for implementation and training.

Despite these challenges and productivity gains, the process of implementation of a PLM solution requires time and effort to adapt the product. This ability to adapt is needed due to the fact that the expertise of every company is what makes this company unique. Capitalizing on the enterprise expertise essentially implies a unique system that comes from the adaptation of a generic PLM system.

Many PLM projects fail to achieve their goals, especially for non-technical reasons. The employees of an organization share common values, corporate culture and social benefits that can be challenged by changing the organization of the company or the introduction of a new IT tool. Change management must take into account these values and and put in place a permanent means of monitoring and identifying group apprehensions in order to, where appropriate, communicate on the stability of the values and current achievements.[1]

The results of a study [2] show that there are either difficulties or rejection in $45 \%$ of cases where PLM systems have been implemented.[3]

Beside the expense of resistance to change, one aspect to consider is the maturity of the company when putting in place and using a PLM system.

Indeed, in addition to an expensive investment, companies must have users willing to use the system. To do this results in an extremely heavy user support to make it adhere to the system, this is what is referred to hereinafter as the management of change

\subsection{Change Management}

Change management is a set of processes employed to ensure that significant changes are implemented in an orderly, controlled and systematic fashion to effect organizational change. It reduces failures and encourages appropriation [4] of new procedures [5]. Approaches to managing change are generally based on following items: participation, communication, and training.

According to Martin [6], change can be seen as a curve including the following steps: doubt, current situation, trigger, project, action plan. 
According to Prochaska and Velicier [7], change is a process operating in cycles, with each cycle comprised of six stages of behavioural change: the pre-intention, intent, preparation, action, maintenance, resolution.

The effectiveness of the implementation of change depends on the quality of the learning process [8] introduced at both individual and team level, as well as on the interaction of both.

Finally, resistance to change is significant. Too often the weight given to the implementation of the technical objective (the software) is disproportionate compared to the effort used in deploying the methodologies of change management when available.

In fact the successfulness of the PLM implementation depends essentially on the way we conduct Change Management.

The PEGASE project (Serious Game Platform Backing and monitoring of change in SMEs / SMIs) [9] defines an environment of support for change management in industrial enterprises with an approach around the Serious Games. The purpose of this project is to develop a real Serious Game platform to help companies to effectively support their staff in adapting to the changes brought about by redesigning their information systems. Some initial actions in this platform game are driven through the integration of real scenarios in industrial processes.
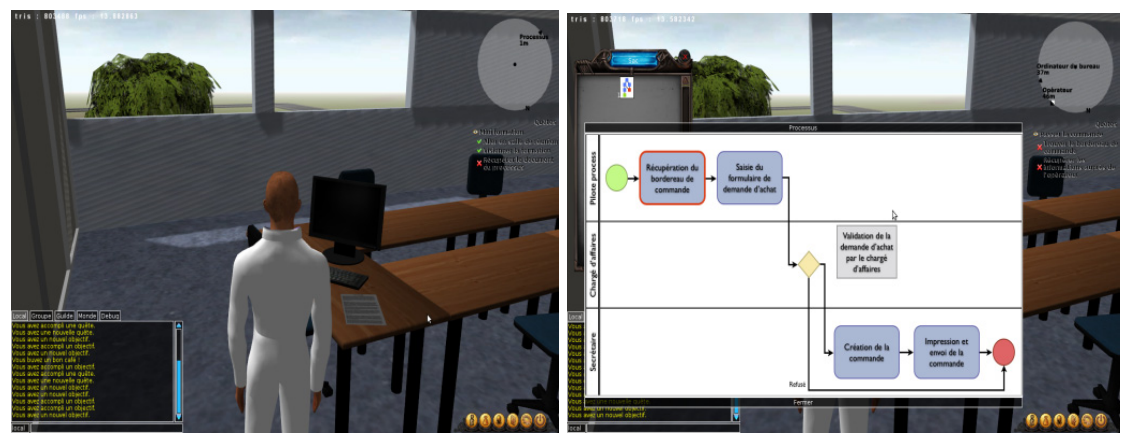

Fig. 1. Screen shot PEGASE: player interacts with its environment
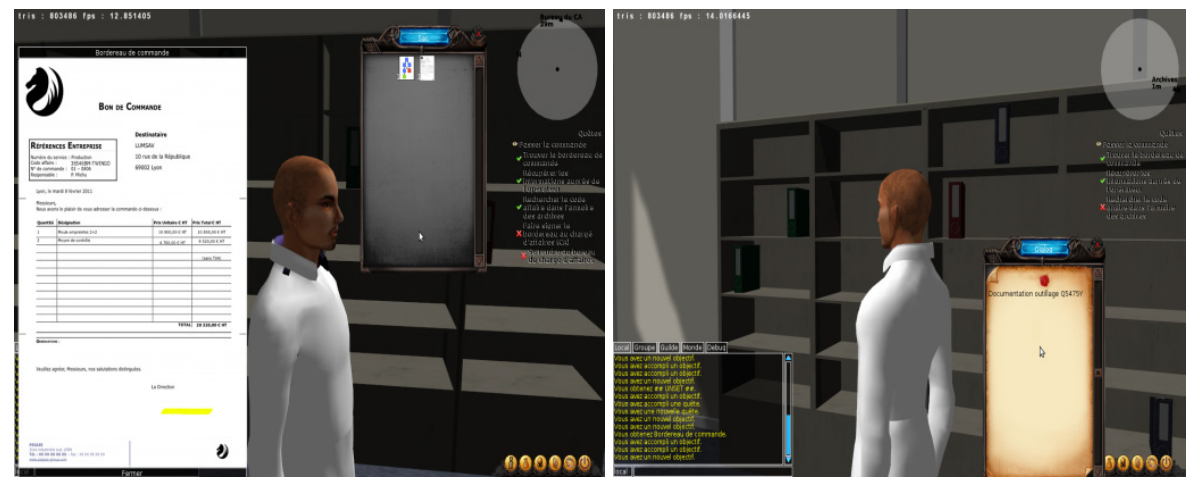

Fig. 2. Screen shot PEGASE: player collects product data 
This project was a success by showing that we can improve change management by using a Serious Game. However, the construction of a Serious Game is very expensive, it does not fit the limited funding of a small and medium sized company.

\section{Approach to Change Management}

\subsection{Bottom Up Approach to Change Management}

With our state of the art analysis, we find that the methods used for change management use a "top-down" approach, in the sense that they take as a starting point, the targeted system to propose methods for supporting the users. Our research introduces a « Bottom up » approach to facilitate the conduct of change. It is therefore necessary to search for elements that are widely known and accepted by the user to support this process.

Today, what we identify as a common utility in all businesses is Windows Explorer and the simple text search box.

Our initiative is thus to work on progressive methods while considering that the success of a PLM project is first and foremost a matter of the Human Machine Interface. The foundations of our approach is a common Human machine interface, simple and therefore natural for most users.

This approach focuses on users and their usual work environment, combining basic features necessary for collaboration: sharing, security, traceability, versioning, rights management.

\subsection{Une Approche Évolutive}

The approach implemented by our research is a comprehensive and evolutionary approach which is divided into 3 phases and smoothly brings the businesses towards better managing their capital expertise, without radically changing the work habits of the users. This methodological approach is progressive and based on what we call the «AudrosBox » approach.

The first phase, collaborative features, is a first level approach allowing for collaboration through Windows Explorer and the simple text search box. On top of these two user interface elements, we extend their functionalities by adding more sophisticated processing which offer powerful functionality for collaborative management. This approach that we call $<<$ plug and play $>>$ targets the fast track approach to collaborative functionalities with minimum training.

The second phase, EDM allows the user to move towards better managing the handling of such data. The use of the Audrosbox system and the habit of working in a collaborative mode induced by the first phase, usually derives from the users newely discovered and more complicated needs in terms of document management. For example when trying to figure out the percentage of copper used in the material specification document given, we will need to be able to manage attributes for each document. 
The third phase, PLM-Product Lifecycle Management, allows users to integrate all of the components for these systems while maintaining a simplified Human Machine Interface. This final phase includes connectors with different CAD systems on the market, the automatic creation of documents, and the management of data dependencies (bill of material).

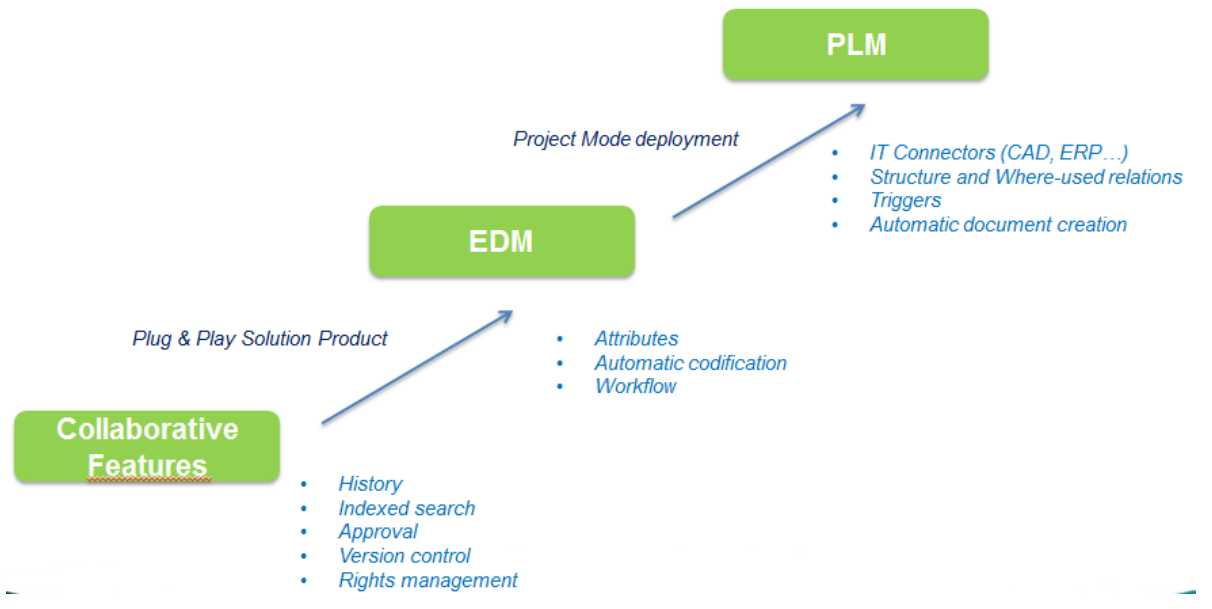

Fig. 3. Methodological approach in 3 phases

\subsection{AudrosBox}

Audrosbox answer to

- the collaborative management of information, mobility and right management

Audrosbox introduces four terminologies,

Repository - it is a context where all the information belongs within this context and obeys the context right managements.

Information - illustrated in the first phase as a file, is created by using instinctively the standard tools of a company.

The engine - which has the function to drive the execution of the tasks needed to make the data compliant on their arrival to the repository.

The cloud or storage space - the information in this cloud space is elected from the repository. 


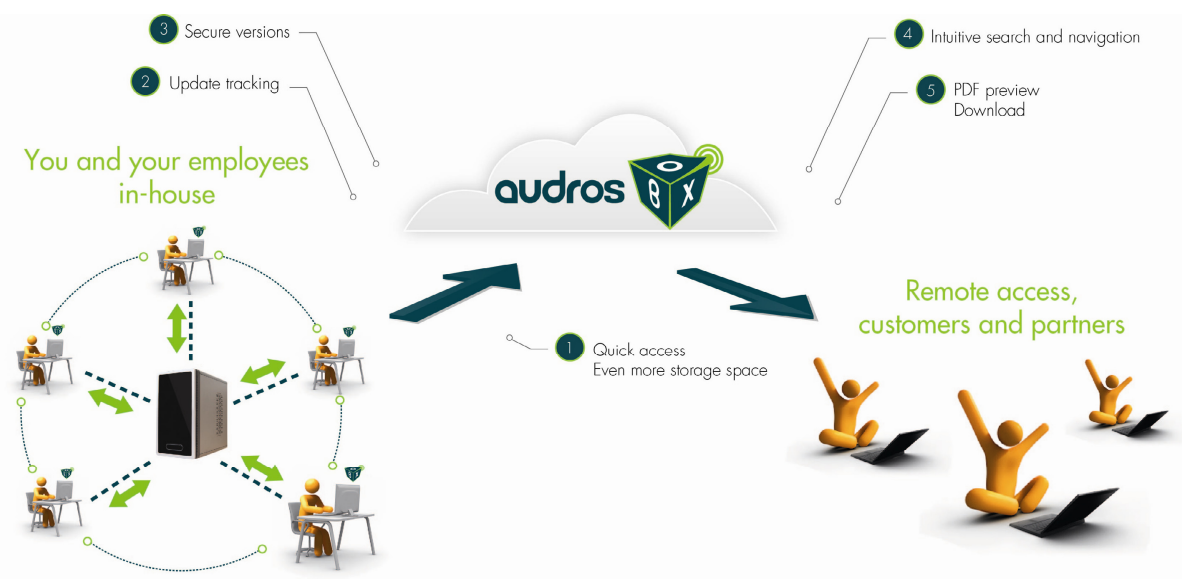

Fig. 4. Audrosbox

\section{$4 \quad$ Functionnality}

The system uses these methods for storing documents onto the repository.

The synchronization of the central system is done by binary comparison of the local repository regarding the same information that is on the remote repository.

The locking systems insure that no information will be updated simultaneously by different users.

The addition of attributes, the detailed business view, allows the user to benefit from the use of these attributes.

This engine allows for the atomization of tasks

\section{Conclusion}

These methods are very promising regarding the number of companies which decide to implement this process. In fact, due to the fast start process with minimum training, the successful introduction of the PLM system to the users can be insured.

We have discovered that we need to enhance our research by means of two topics:

- The simple text search needs to be more powerful and should not only deliver as an answer to the texts we find in the index of the documentation, but also the implementation of the semantic search should enhance this functionality.

In the $3 \mathrm{dr}$ phase, PLM level, it is very important to retain the malleability of the model. This is why we have begun this research 


\section{References}

1. Bissay, A.: Du deployment d'un système PLM vers une integration des connaissances, Thèse de doctorat, Université Lyon 2 (2010)

2. Kadiri, S., Pernelle, P., Delattre, M., Bouras, A.: Current situation of PLM systems in SME/SMI: Survey's results and analysis. In: PLM 2009, Bath, England (2009)

3. Kadiri, S.: Management des processus collaboratifs dans les systèmes PLM, Thèse de doctorat, Université Lyon 2 (2009)

4. Gélinier, O.: Stratégies de l'entreprise et motivation des hommes, éd. d'Organisation (1990)

5. Savall, H., Zardet, V.: Ingénierie Stratégique du Roseau, Economica (1995)

6. Martin, J.: La Conduite du Changement, Université de Strasbourg (2009), http: //univrcms.ustrasbg.fr/depotcel/DepotCel/514/ueop0910s1/ SMQ_ConduiteChangement.ppt

7. Prochaska, J.O., Velicer, W.F.: The transtheoretical model of health behavior change. American Journal of Health Promotion (1997)

8. Argyris, C.: On Organizational learning. Blackwell Business (1992)

9. Bissay, A., Cheballah, K., Zrouki, M., Pernelle, P.: PEGASE: a platform tool to help change management support during the implementation of a PLM system in an industrial company. In: PLM 2011, Eindoven, Pays Bas (2011) 